\title{
The bithorax complex: control of segmental identity
}

\author{
Mark Peifer, François Karch, ${ }^{1}$ and Welcome Bender \\ Department of Biological Chemistry, Harvard Medical School, Boston, Massachusetts 02115 and ${ }^{1}$ Department of Animal \\ Biology, University of Geneva, 1224 Chêne-Bougeries, Geneva, Switzerland
}

Like many organisms, the fruit fly, Drosophila melanogaster, has a segmented body. Subdivision into segments is controlled by the segmentation genes (Nüsslein -Volhard and Wieschaus 1980), while the homeotic genes determine segmental identity. Homeotic mutations alter the identity of particular segments, transforming them into copies of other segments. The homeotic genes cluster in two complex loci. The Antennapedia complex (Kaufman et al. 1980) is responsible for differentiation of the head, and the first thoracic and anterior second thoracic segments ( $\mathrm{T} 1$ and aT2), while the bithorax complex (BX-C; Lewis 1978) is responsible for differentiation of the remaining thoracic (posterior $\mathrm{T} 2$, and $\mathrm{T} 3$ ) and eight abdominal segments $\mathrm{Al}-\mathrm{A} 8$ ). In the absence of both complexes, the embryo develops as a series of identical thoracic segments. Thus, the ANT-C and the BX-C are apparently the only loci that receive (or remember) the positional information that initially numbers the segments.

The genetic analysis of the BX-C has been reviewed recently (Duncan 1987), so we will confine this article to a model of BX-C function. This model invokes regulatory regions of an unprecedented size. Complex cis-regulation and trans-regulatory interactions between the different BX-C products produce segments, each of which is a mosaic of cells expressing different homeotic proteins. The unique mosaic of pattern elements produces the distinctive structures of a particular segment. This model explains much of the genetic and molecular complexity of the BX-C.

\section{Nomenclature}

We begin by clarifying two points of nomenclature. Recently it has been proposed that BX-C regulation occurs not through segments but through parasegments, each composed of the posterior compartment of one segment through the anterior compartment of the next segment (Hayes et al. 1984; Struhl 1984; Martinez-Arias and Lawrence 1985). Thus, for example, parasegment 5 (PS 5 ) comprises pT2 and aT3, while parasegment 6 (PS 6) comprises $\mathrm{pT} 3$ and aAl. This view has substantial experimental support, and we will use it to describe many of the observations.

Two nomenclatures exist in the literature for describing the mutations of the BX-C (Karch et al. 1985; Sanchez-Herrero et al. 1985). We will use in this review, and in our subsequent work, a compromise terminology, based on the molecular data currently available. We will call mutations that affect protein-coding units Ultrabithorax (Ubx), abdominal-A (abd-A), and Abdominal$B(A b d-B)$, while mutations that affect their regulation retain the designations anterobithorax $(a b x)$, bithorax $(b x)$, bithoraxoid $(b x d)$, postbithorax $(p b x)$, and infrabdominal-2 (iab-2) to infrabdominal-8,9 (iab-8,9) (Duncan 1987). The $a b x$ and $b x$ mutations overlap in phenotype, as do the $b x d$ and $p b x$ mutations. Because of the overlap, we will often discuss these as the $a b x / b x$ and $b \times d / p b x$ mutational classes.

\section{Very large regulatory regions}

One of the most striking features of the BX-C is its size; it covers more than $300 \mathrm{~kb}$ (Bender et al. 1985; Karch et al. 1985). Genetic screens have revealed three classes of lethal mutations in the BX-C: $U b x, a b d-A, A b d-B$ (Sanchez-Herrero et al. 1985; Tiong et al. 1985). These mutations define three transcription units; all three contain homeo boxes (Regulaski et al. 1985), and probably encode families of DNA binding proteins (Laughon and Scott 1984; Gehring 1987), produced by alternate splicing. Transcriptional analysis is still incomplete, but these proteins probably only require $10-20 \mathrm{~kb}$ of coding sequence considered together. The three transcription units may well be the only protein-coding units in the BX-C; this is supported by the report that the embryonic lethal phenotype of an $U b x, a b d-A, A b d-B$ triple point mutant is identical to that of a deletion of the entire BX-C (Casanova et al. 1987). We note that other RNA products are transcribed from the complex (Lipshitz et al. 1987), but most of these apparently do not code for proteins, and they have no known functions.

Subtracting the DNA required to encode the three protein families still leaves more than $250 \mathrm{~kb}$ in the complex. Unlike mammalian genes, in which large introns are commonplace, most "simple" Drosophila genes span less than $10 \mathrm{~kb}$. We believe that the "excess" DNA in the BX-C comprises regulatory regions of great size and complexity. These regulatory regions affect transcription from upstream, downstream, or within the introns of transcription units; in some cases the regulatory sequences are more than $50 \mathrm{~kb}$ from the transcription start site.

Figure 1 outlines the molecular organization of the BX-C (Bender et al. 1983; Karch et al. 1985; Duncan 1987). The three transcription units encoding protein (Ubx, abd-A, and $A b d-B)$ are indicated below the scale. Above the scale are the putative regulatory regions, as 
Peifer et al.

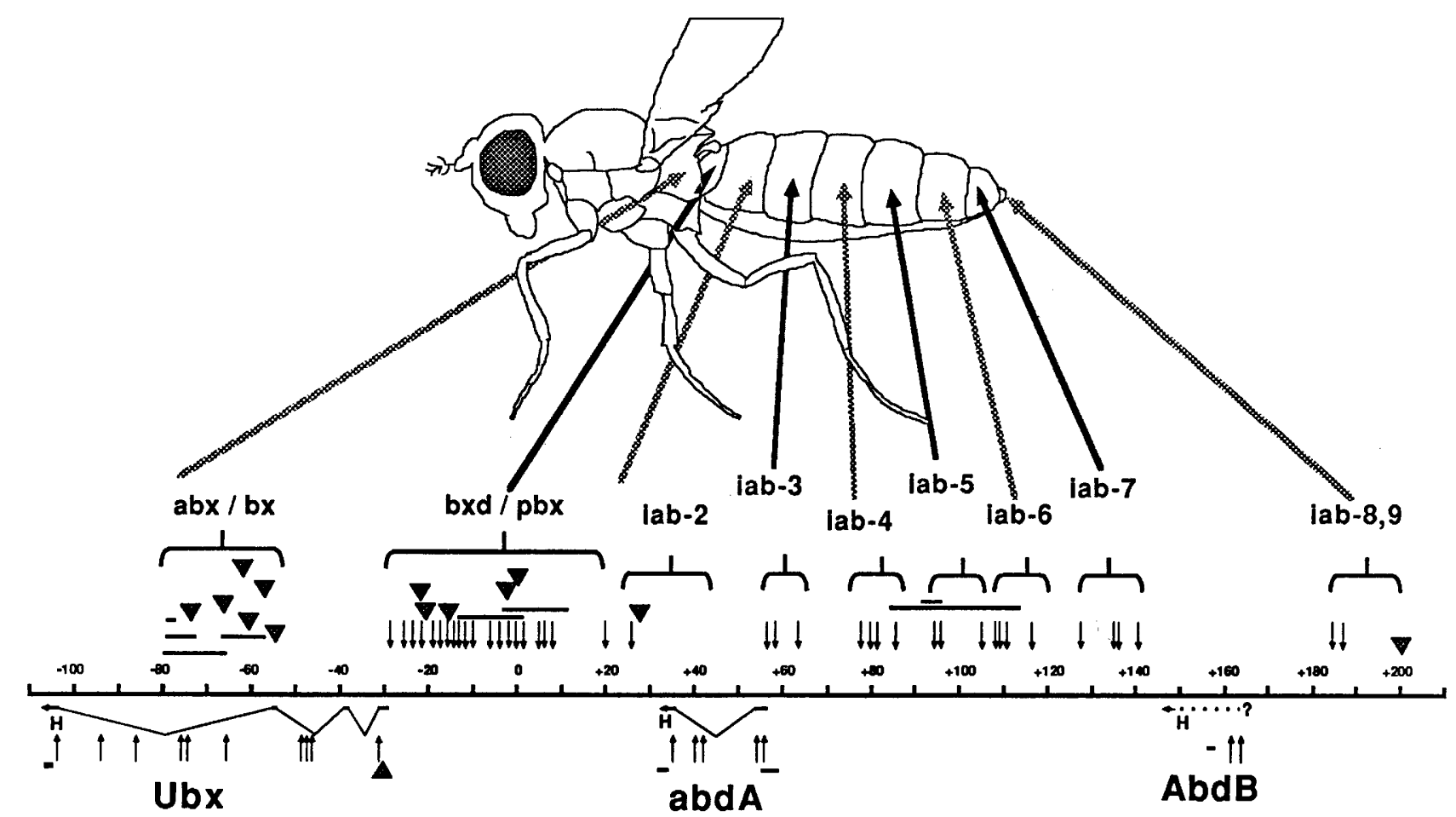

Figure 1. Mutations in the bithorax complex. The continuous line shows the $300-\mathrm{kb}$ DNA map of the BX-C. Below the line are shown the three transcription units encoding homeotic proteins. All are transcribed from right to left, and all include a homeo box sequence (designated $\mathrm{H}$ ) near their $3^{\prime}$ ends. The structure of the $A b d-B$ transcription unit is still uncertain. Below the transcription units are shown mutant lesions which inactivate that transcript. Arrows indicate rearrangement breakpoints, horizontal bars indicate deletions, and triangles indicate insertions of mobile elements. Above the DNA line are shown lesions of regulatory mutations. These are grouped according to the most anterior parasegment of the fly which they affect, and the arrows indicate the portion of an adult female fly corresponding to each of these parasegments.

defined by the indicated mutations. While mutations in a specific regulatory region affect more than one parasegment, we have indicated only the most anterior parasegment of the fly which such a mutation affects. This is typically the parasegment in which the mutation's effect is most striking. The lesions of a given class cluster in a relatively small part of the BX-C, and different classes do not overlap on the DNA. As initial recombinational mapping suggested (Lewis 1978), there is an almost perfect correspondence between the order of the mutant lesions and that of the segments that they affect.

The distinction between protein-coding and regulatory regions explains the complex complementation among the loci of the BX-C. The pattern of complementation in the BX-C can be organized around $U b x, a b d-A$, and $A b d-B$. Ubx mutations result in complete transformation of PS 5 and PS 6 to PS 4 , and have more subtle phenotypic effects on pattern in the abdomen (Lewis 1978; Hayes et al. 1984). Different subsets of the $U b x$ transformation are seen in the mutational classes $a b x /$ $b x$ and $b x d / p b x . a b x / b x$ mutations partially complement $b x d / p b x$ mutations, but both classes fail to complement $U b x$ (Lewis 1978). These mutations have been described as affecting independent functions, each of which is "cis-inactivated" by a Ubx mutation. It is simpler to suggest that $a b x / b x$ and $b x d / p b x$ mutations affect distinct regulatory regions responsible for the ac- tivation of $U b x$ protein in particular parts of the fly. A mutation affecting the coding sequence for the $U b x$ protein (a $U b x$ mutation) would fail to complement all of the regulatory mutations which affect $U b x$ expression. In contrast, mutations in distinct regulatory regions would be expected to complement each other. Recent work with antibodies to $U b x$ protein confirms that $a b x$, $b x, b x d$, and $p b x$ mutations all affect the pattern of $U b x$ expression (see below).

A similar argument explains the complementation patterns of mutations affecting the abdomen. $a b d-A$ mutations affect PS 7 to PS 13 (segments A2-A8) (Morata et al. 1983). When tested in trans, abd-A mutations fail to complement $i a b-2, i a b-3, i a b-4$, and, in some cases, iab-5 mutations (Karch et al. 1985). Abd-B mutations (which when homozygous affect PS 10 to PS 14 (Sanchez-Herrero et al. 1985; Tiong et al. 1985) fail to complement $i a b-5,-6,-7$, and $-8,9$ (Karch et al. 1985). Once again, we postulate that these complementation interactions are explained by the fact that a mutation in a protein product will fail to complement all mutations that affect expression of the protein.

All mutations affecting the thorax (Ubx, etc.) fully complement mutations affecting the abdomen $(a b d-A$, $A b d-B$, and iab-2-iab-8,9; Struhl 1984). Complementation between abdominal mutations is more complex. Indirect evidence from genetic complementation (Karch et 
al. 1985) and from examination of $U b x$ and $a b d-A$ expression (F. Karch and W. Bender, unpubl.) suggests that the regulatory regions $i a b-2$ to $i a b-7$ may all regulate to some extent both $a b d-A$ and $A b d-B$. Definitive evidence will come from the description of $a b d-A$ and $A b d-B$ protein expression in mutant animals.

\section{"Mosaic" segments}

The fine tuning mediated by these large regulatory regions results in an intricate pattern of gene expression, as revealed by in-situ hybridization to $U b x$ RNA and by immunological detection of $U b x$ protein (Akam and Martinez-Arias 1985; White and Wilcox 1984, 1985a; Beachy et al. 1985). For example, in the germ-band retracted embryo, the pattern of $U b x$ expression is rela- tively simple in PS 5. In PS 6 new cells express $U b x$ and others change in intensity relative to PS 5 . Ubx expression is progressively diminished in more posterior parasegments. The reason for the diminution of $U b x$ expression in the abdominal segments is the activation of the other two proteins of the BX- C, $a b d-A$ and $A b d-B$. Either of these two proteins can repress $U b x$ (Struhl and White 1985). The expression of $a b d-A$, as assayed by antibody staining (F. Karch and W. Bender, unpubl.), is similar in many ways to that of $U b x$. Expression in the germ-band retracted embryo is first activated in PS 7, it increases in more posterior parasegments, and then is diminished in PS 13 and PS 14 , presumably by the product of $A b d-B$.

A simplified and schematic representation of these patterns is presented in Figure 2, along with a model of

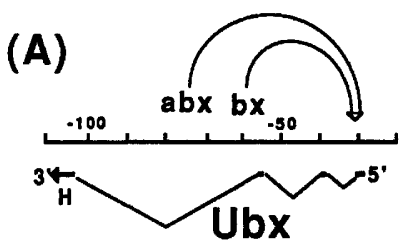

\section{Parasegments:}

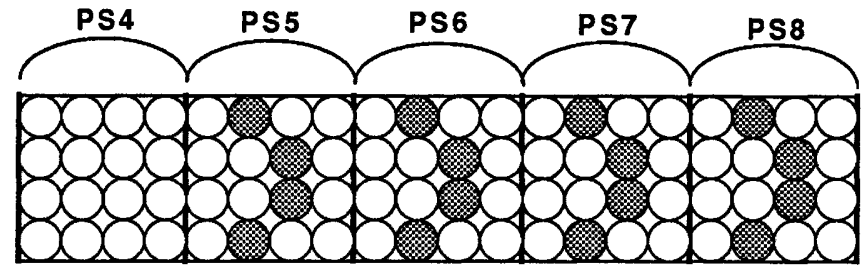

(B)
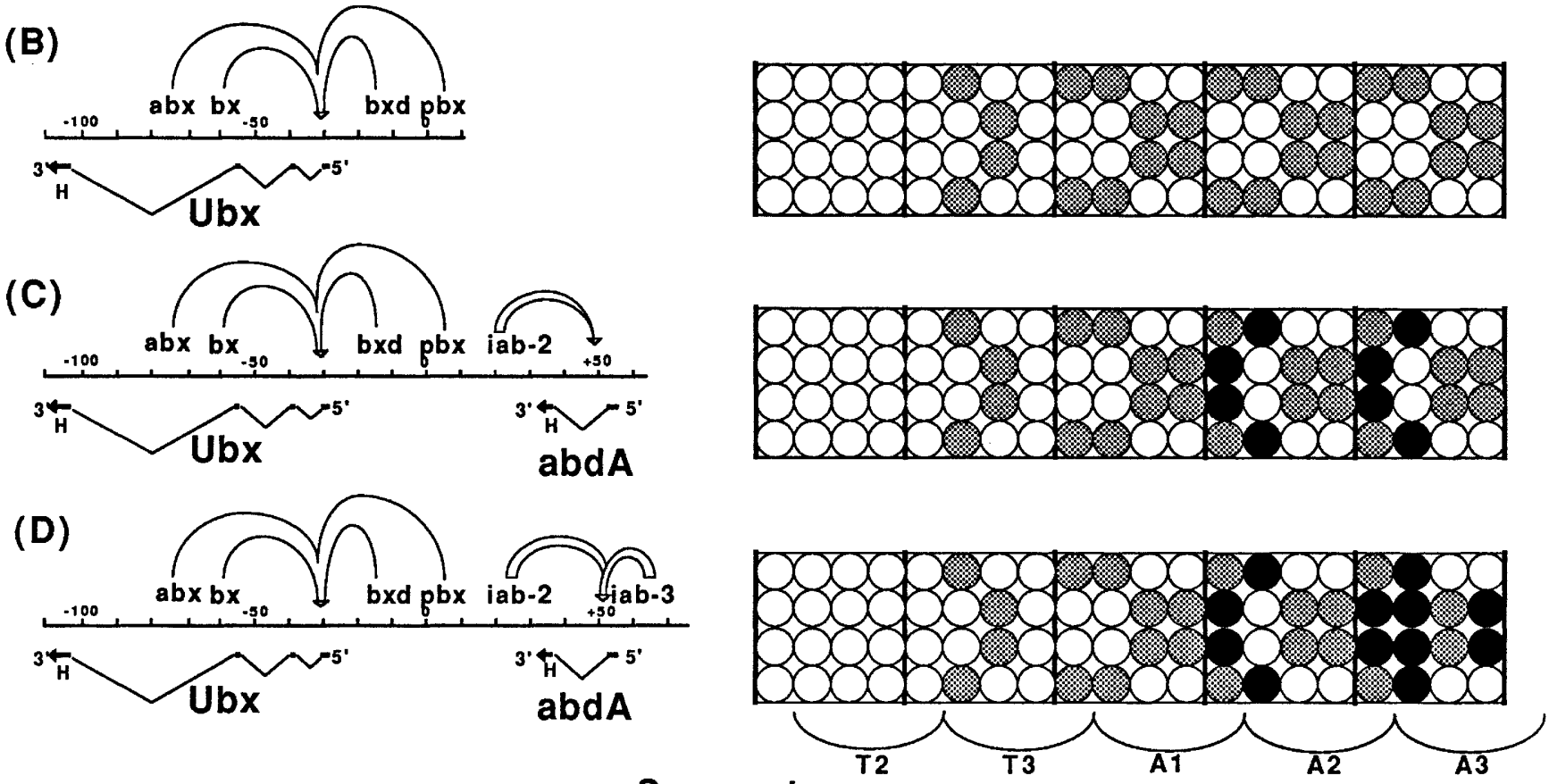

Segments:

Figure 2. Buildup of mosaic patterns. On the left are diagrams of successively larger portions of the BX-C, with transcription and regulatory units marked. On the right are cartoons of a sequence of five parasegments, with circles representing individual cells of the ectoderm and nervous system. The boundaries of two thoracic and three abdominal segments are shown at the bottom, to indicate the relation between segmental and parasegmental boundaries. Circles filled in grey represent cells expressing $U b x$ protein, and those in black represent cells expressing $a b d-A$ protein. These cartoons are based primarily on the patterns of $U b x$ and $a b d-A$ protein in embryos with deletions extending from the right end of the BX-C. The patterns are not meant to represent the actual pattern of expression. (A) The leftmost $80 \mathrm{~kb}$ of the BX-C contains only the $a b x / b x$ regulatory domain and the $U b x$ transcription unit. $U b x$ first appears in PS 5, and repeats in the same pattern in the more posterior parasegments. $(B)$ When $130 \mathrm{~kb}$ is present, the $b x d / p b x$ regulatory domain turns on $U b x$ protein in additional cells in PS 6, and the corresponding cells express $U b x$ in the more posterior parasegments. $(C)$ With $170 \mathrm{~kb}$ present, $a b d-A$ protein is now expressed in PS 7 and more posterior parasegments, under control of $i a b-2$. When $a b d-A$ protein is made in a cell that would otherwise express $U b x$ protein, expression of $U b x$ is repressed. $(D)$ With 190 $\mathrm{kb}$, abd-A protein is expressed in additional cells in PS 8, and in more posterior parasegments. 
Peifer et al.

how they are produced. Each circle represents a cell in the indicated parasegment. Generally, a cell expresses strongly only one homeotic product; in this representation we are only outlining the expression of two of these products, $U b x$ (grey circles) and $a b d-A$ (filled circles). While the pattern of expression in a wild-type embryos is complex, it can be built up by postulating a relatively simple set of cis- and trans- regulatory interactions. If one examined an embryo carrying only the $U b x$ transcription unit along with its associated $a b x / b x$ regulatory region, one would see the pattern of expression in Figure 2A. Expression of $U b x$ would be activated in PS 5, and this same pattern would be seen in all more posterior parasegments. When one adds to this the $b x d / p b x$ regulatory region, one obtains the more complex pattern in Figure 2B. The regulatory elements of the $b x d / p b x$ region enhance expression of $U b x$ in PS 6, turning it on in new cells and increasing its expression in others. This pattern is once again repeated in all more posterior parasegments.

The addition of the $a b d \cdot A$ transcription unit, along with its associated regulatory sequences in $i a b-2$, results in two further changes to the pattern (Fig. 2C). First, $i a b-2$ activates $a b d-A$ expression in certain cells of PS 7 , and the more posterior parasegments. Second, the $a b d-A$ product thus produced reduces the expression of $U b x$, when $a b d-A$ and $U b x$ appear in the same cell. The effect of the $i a b-3$ regulatory region is additional elaboration of the $a b d-A$ pattern in PS 8 and more posterior parasegments (Fig. 2D). Addition of the other regulatory elements of $a b d-A$, along with the contribution of $A b d-B$, and its associated regulatory elements, results in the complex pattern seen in the wild type fly. It is possible that Figure 2 should also indicate some cells expressing $A b d-B$ in PS 7 and PS 8. We have also omitted, for

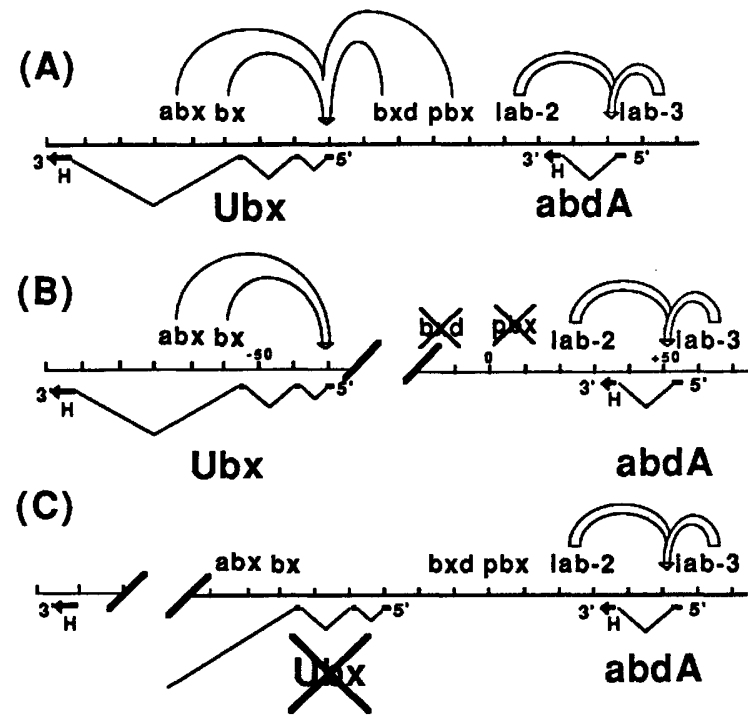

reasons of simplicity, mention of the Antennapedia protein. This protein appears in cells of PS 4-PS 12; although it is turned off in many cells of PS 5-PS 12 by products of the BX-C (Carroll et al. 1986; Wirz et al. 1986). Thus each parasegment is mosaic in nature, containing different cells expressing several different homeotic proteins.

\section{Interpretation of mutant phenotypes}

As can be seen in Figure 3, the loss of a given protein results in major changes in the mosaic pattern of expression in a number of parasegments. These effects stem both from the loss of that protein from the pattern, and from the expansion of the expression of other homeotic products more posteriorally, due to the removal of trans-repression. This is clearly illustrated by the example of $U b x$. In its absence, the pattern of homeotic gene expression in PS 5 and PS 6 is dramatically altered (Fig. 3C). When $U b x$ expression is abolished, the expression of Antennapedia protein (not shown in the figures) expands into PS 5 and 6 , in a pattern identical to that in PS 4 (Carrol et al. 1986). As a result, PS 5 and PS 6 are transformed into copies of PS 4 . Such a severe transformation is lethal. In addition to these dramatic effects, loss of $U b x$ has a more subtle effect on the more posterior parasegments. For example, Lewis (1978) noticed that in $U b x$ mutants, thoracic sense organs of the larva, called ventral pits, are now found on the abdominal segments as well. This is also consistent with the model, since abdominal parasegments are mosaics in which a small but significant number of cells express $U b x$. Thus, in one of the abdominal parasegments, $U b x$ functions in some cells to suppress thoracic sense organs, while other

Parasegments:
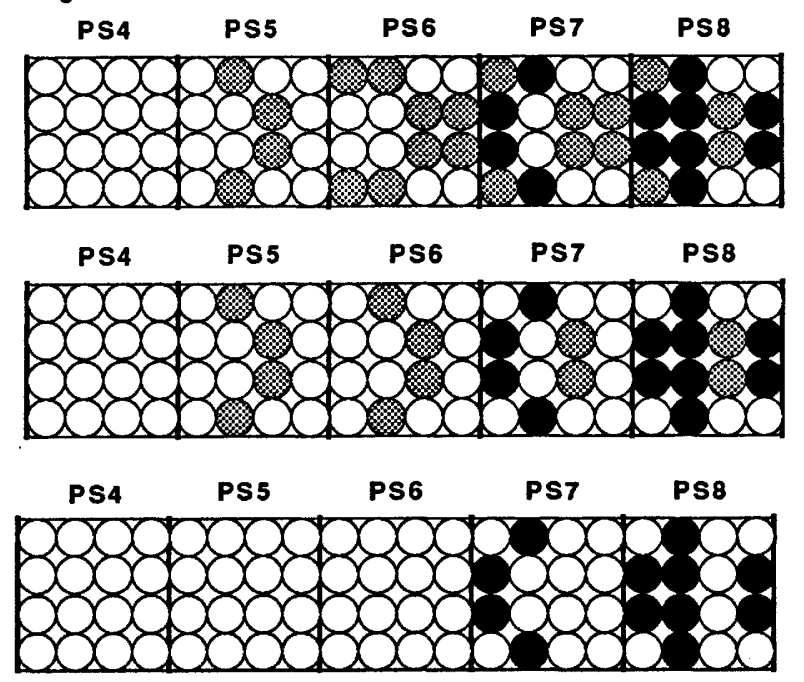

Figure 3. Mosaic patterns in $U b x$ and $b x d$ mutations. The diagrams are analogous to those in Fig. 2. (A) The wild-type pattern (as in Fig. 2D). (B) An extreme $b x d$ mutation, a rearrangement that breaks the chromosome just upstream of the $U b x 5^{\prime}$ end, and separates the whole $b x d / p b x$ regulatory region from the $U b x$ transcription unit. The $U b x$ protein pattern is dramatically effected in PS 6, and also changed in more posterior parasegments. (C) An extreme $U b x$ mutation (a rearrangement that breaks within the $U b x$ transcription unit) removes $U b x$ protein in all segments. PS 5 and PS 6 are most obviously affected, but the pattern in more posterior parasegments is also changed. 
sense organs appear from mutational analysis to arise from cells expressing $a b d-A$.

Regulatory mutations cause more limited phenotypic effects. These mutations alter expression of a homeotic protein in particular cells in particular parasegments. Usually a class of mutations affects one parasegment most dramatically, transforming it toward the immediately more anterior parasegment; each class also has more subtle effects on more posterior parasegments. This is easiest to visualize in the case of the $b x d / p b x$ mutations (Fig. 3B). These mutants have dramatic effects on PS 6, resulting in transformation to PS 5 (pT3 and $\mathrm{aAl}$ toward $\mathrm{pT} 2$ and $\mathrm{aT} 3$ ). In addition, more subtle changes are seen in the abdominal segments, including effects on larval sense organs (Lewis 1978). The effect of $b x d / p b x$ mutations on $U b x$ expression is consistent with this phenotype: they lower Ubx expression dramatically in PS 6 (Beachy et al. 1985; White and Wilcox $1985 \mathrm{~b}$ ). In the case of an extreme bxd mutation, PS 6 has nearly the same pattern of $U b x$ expression as PS 5 ; this residual expression is presumably directed by $a b x / b x$ (W. Bender, in prep.). Strong $b x d / p b x$ mutations also have more mild effects on $U b x$ pattern in the abdomen.

Mutations in other regulatory regions show a similar correlation between effects on expression and phenotypic consequences. In $a b x / b x$ mutants, PS 5 is transformed into PS 4 (Casanova et al. 1985; Peifer and Bender 1986); $U b x$ expression in $a b x / b x$ mutants is reduced in PS 5 (Cabrera et al. 1985; White and Wilcox 1985b). The iab mutants have analogous phenotypic effects on the abdominal parasegments. Preliminary analysis suggests that the $i a b$ mutations alter the expression of $a b d-A$ in the expected parasegment (F. Karch and W. Bender, unpubl.); the $i a b$ mutations presumably also effect expression of $A b d-B$.

\section{Domains of regulation}

The regulatory regions of the $\mathrm{BX}-\mathrm{C}$ are arranged on the chromosome in the same order as the anteriormost affected parasegments are arranged on the body axis (Fig. 1). In each more posterior parasegment, new regulatory domains are activated sequentially along the chromosome. Thus, in PS 5 , only the $a b x / b x$ region is active. In PS 6, it remains active, while the next adjacent regulatory domain, $b x d / p b x$ is also activated. This activation continues, with each more posterior parasegment seeing the activation of the next adjacent regulatory region. The concept of sequential activation of BX-C functions was part of the original model of the BX-C proposed by Lewis (1978). In its original formulation, Lewis proposed that new gene products are turned on in each successive segment. Our reinterpretation invokes, instead, the activation of successive regulatory regions, producing a unique pattern of the three gene products in each successive segment.

The evidence suggests that each regulatory region contains several elements which together specify the pattern of protein expression. Mutations in the $b x d / p b x$ region most clearly illustrate this. Mapping revealed a series of rearrangement breaks which span the entire 50 $\mathrm{kb}$ upstream of $U b \mathrm{~b}$. The closer a rearrangement break is to the $5^{\prime}$ end of $U b x$, the more severe its $b x d$ phenotype (Bender et al. 1985). The $b x d$ mutations have also been examined for expression of $U b x$ protein, using $U b x$ antibody (W. Bender, in prep.). The more bxd DNA removed, the fewer cells in PS 6 express $U b x$. Together, phenotypes and analysis of $U b x$ protein patterns suggest that there are multiple, independent elements (perhaps equivalent to enhancers) present in the $b x d / p b x$ region which activate $U b x$ expression in PS 6 and the more posterior parasegments.

The standard definition of an enhancer includes the ability to function in a variety of positions with respect to the transcription start. If the regulation of the BX-C was accomplished only by such enhancers, there would be no need for the apparent clustering of elements required in a particular set of parasegments, like those in the $b x d / p b x$ region. A probable reason for this clustering is illustrated by the informative mutants $C b x^{1}$ and $p b x^{1}$. In $C b x^{1} p b x^{1}$ animals, a piece of DNA from upstream of $U b x$ is excised, and inserted in the opposite orientation in the second intron (Fig. 4; Bender et al. 1983). The deletion alone $\left(p b x^{1}\right)$ transforms posterior haltere to posterior wing (Fig. 4). The insertion, $C b x^{1}$, does the opposite, transforming wing to haltere, and also rescues the phenotype of the deletion (Lewis 1963). These mutations cause the expected alteration in $U b x$ expression. The deletion abolishes $U b x$ expression in the posterior haltere disc, while the insertion activates $U b x$ ectopically in the posterior wing disc (Fig. 4; Cabrera et al. 1985; White and Akam 1985; White and Wilcox 1985b).

These phenotypes are most easily explained in terms of cell-specific enhancers and parasegment-specific DNA domains. The DNA deleted in $p b x^{1}$ presumably contains enhancers responsible for expression of $U b x$ in particular cell types (the D cells in Fig. 4). These enhancers are normally active in the D cells in PS 6, which give rise to the posterior haltere. The deletion of these enhancers in $p b x^{1}$ leads to the loss of expression of $U b x$ in the $\mathrm{D}$ cells, and the posterior haltere is transformed to posterior wing (Fig. 4). If these enhancers were responsive both to cell type and to parasegmental identity, moving them upstream of $U b x$ to downstream would probably not effect their function. In fact, this transposition leads to the dramatic dominant phenotype of $C b x^{1}$. In their new position, the enhancers are activated in the D cells of PS 5 as well as PS 6, transforming the posterior wing into posterior haltere (Fig. 4).

This surprising result can be accommodated by postulating that parasegmental address is conferred by the DNA domain in which an enhancer resides. Each regulatory locus (e.g., $a b x / b x$ or $b x d / p b x)$ would comprise a DNA domain (Fig. 4). Each domain, in response to genes involved in specifying the anterior-posterior axis, would be accessible to trans-regulatory products in some parasegments but not others. For example, in PS 5 the $a b x / b x$ domain (light shading in Fig. 4) would be made accessible to trans-regulatory factors, while the other domains (e.g., $b x d / p b x$; heavy shading in Fig. 4) would 
Peifer et al.

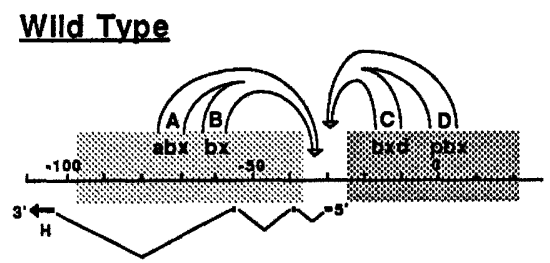

Ubx

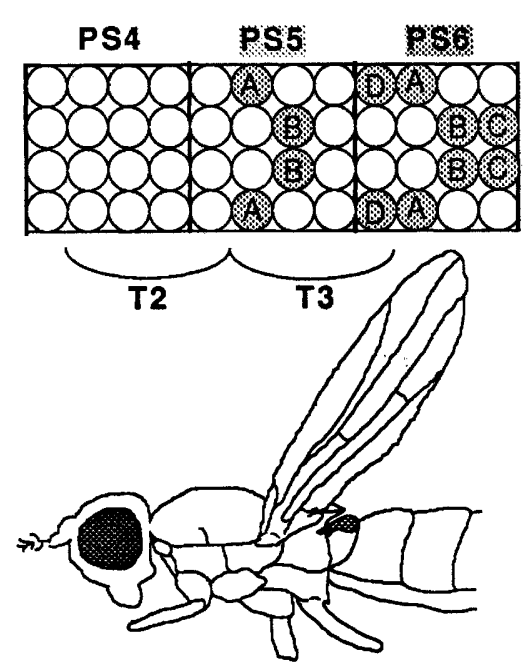

pbx

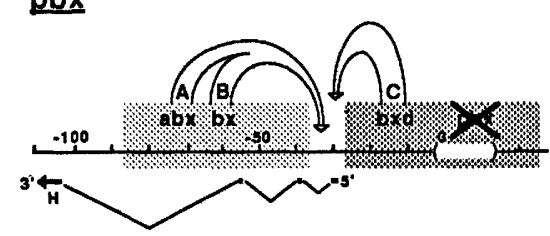

Ubx

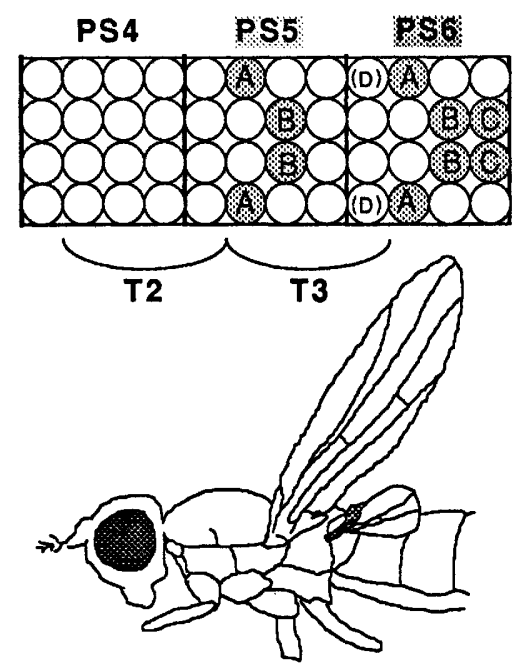

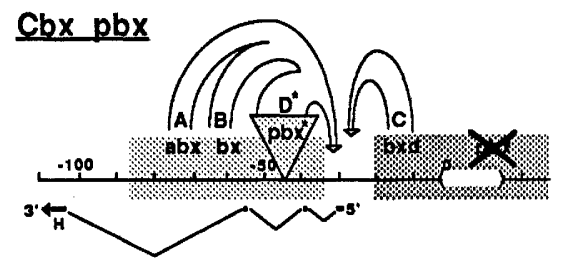

Ubx
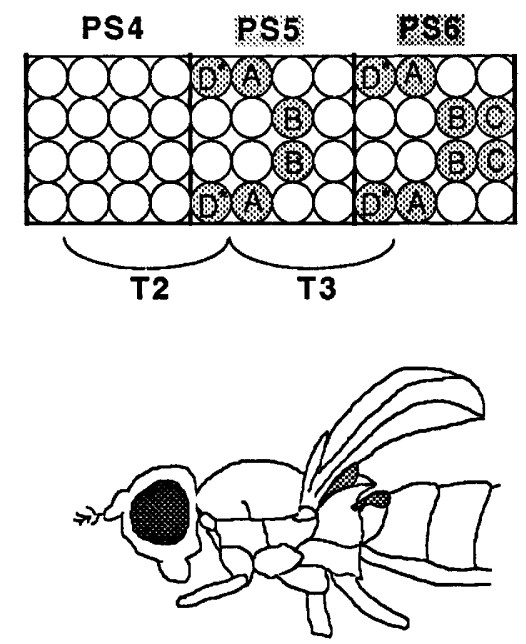

Figure 4. Patterns and transformations in $p b x$ and $C b x p b x$ genotypes. Regulatory domains and $U b x$ expression patterns are diagramed as in Fig. 2, and the phenotypes of adult flies are diagramed at the bottom. Haltere tissue is shaded grey in the drawings of the flies. The regulatory domains are distinguished by different shadings; the $a b x / b x$ domain is active in PS 5 , and the $b x d / p b x$ domain is active in PS 6. The capital letters, A-D, indicate individual enhancer-like elements specifying particular cells in the animal; these cells are lettered in the cartoons of the $U b x$ protein patterns. The $p b x$ mutation is a deletion of $17 \mathrm{~kb}$ from the PS 6 regulatory domain, and the $C b x$ mutation is an insertion of that same $17-\mathrm{kb}$ segment in the PS 5 domain. This 17-kb DNA segment normally turns on $U b x$ protein in PS 6 in cells that will generate the posterior haltere (the circles labeled D). In the animal with the 17-kb segment deleted, $U b x$ protein is not made in the $\mathrm{D}$ cells, and the posterior haltere is transformed to wing. In the $C b x p b x$ animal, with the 17 $\mathrm{kb}$ transposed to the PS 5 domain, Ubx is aberrantly expressed in the D cells of PS 5, as well as PS 6 . The D cells in PS 5 normally generate posterior wing, but now, because of $U b x$ expression, they make posterior haltere.

be closed. We envision within the $a b x / b x$ domain numberous enhancers (represented by A and B in Fig. 4), each reading the activity state of genes specifying cell identity, perhaps by juxtaposed binding sites for a number of DNA-binding proteins. Each enhancer would activate $U b x$ in a particular cell or set of cells in PS 5 (the A and B cells of Fig. 4, respectively), as well as in the corresponding cells of more posterior parasegments. In PS 6, the $b x d / p b x$ domain is also opened, in response to an altered set of positional cues. Within this domain are a different set of enhancers (C and $D$ in Fig. 4$)$, which activate $U b x$ in a new pattern of cells (the $C$ and $D$ cells). This pattern is added in PS 6 to that imposed by $a b x / b x$. Our model requires that the $5^{\prime}$ end of $U b x$ be accessible in all cells of PS 5-PS 13, but it is only activated in concert with one of the regulatory regions. In each more posterior parasegment, the next adjacent DNA domain would be opened up $(i a b-2, i a b-3$, etc.). Thus the order on the chromosome reflects the order of opening of DNA domains, and this order may be mechanistically required for appropriate function.

This model provides an explanation of $C b x^{1}$. When the enhancers from the $p b x$ region were transposed, they were moved from a domain active in PS 6 to a domain active in PS 5. They now turn on $U b x$ expression in the D cells of PS 5 (Fig. 4), resulting in the transformation. Since this domain would also be open in more posterior segments, the insertion should rescue the phenotype of the deletion, a prediction that is borne out.

\section{Conclusion}

Since Lewis proposed his model of BX-C function, there have been many advances in the characterization of this region. This analysis has supported most features of the Lewis model, which can now be stated in molecular terms. Lewis (1978) initially envisioned activation of a new gene product in each segment. The combinatorial action of the spectrum of products active in a particular segment determined its identity. It now seems likely that three major groups of related protein products are encoded by the BX-C, each acting within a subset of the fly's segments. Discrete genetic units exist, however, which are sequentially activated in each parasegment. These units function as transcription regulatory regions. The complex cis-regulation that they mediate and the trans-interactions between the different homeotic genes result in very intricate patterns of expression, both be- 
tween and within parasegments. There are not segmentwide strips of expression of different BX-C gene products. Instead, each parasegment is a mosaic of cells expressing different homeotic proteins. Under the direction of these proteins, different cells adopt different fates, yielding the complex array of pattern elements which characterizes a given parasegment.

Large regulatory regions may not be an unusual feature of genes regulating pattern formation. Several other genes, including those of the engrailed locus (Kuner et al. 1985), the decapentaplegic complex (Gelbart et al. 1985), and the ANT-C (Scott et al. 1983), have large regions which may be involved in regulation. The uniqueness of the BX-C is the higher order organization of its DNA domains. This machinery enables the BX-C to specify a complex and unique pattern of protein expression in each of the successive segments of the thorax and the abdomen, regulating with the products of only three genes the segmental differentiation of nine segments.

\section{Acknowledgments}

We thank Ed Lewis, Ian Duncan, and members of our laboratories for helpful discussions, and Gines Morata for sharing results before publication. We are also indebted to Alisòn DeLong, Fred Winston, and Anne Happel for numerous helpful suggestions on the manuscript.

\section{References}

Akam, M.E. and A. Martinez-Arias. 1985. The distribution of Ultrabithorax transcripts in Drosophila embryos. EMBO $I$. 4: $1689-1700$.

Beachy, P.A., S.L. Helfand, and D.S. Hogness. 1985. Segmental distribution of bithorax complex proteins during Drosophila development. Nature 313: 545-551.

Bender, W., B. Weiffenbach, F. Karch, and M. Peifer. 1985. Domains of cis-interaction in the bithorax complex. Cold Spring Harbor Symp. Quant. Biol. 50: 173-180.

Bender W., M. Akam, F. Karch, P.A. Beachy, M. Peifer, P. Spierer, E.B. Lewis, and D.S. Hogness. 1983. Molecular genetics of the bithorax complex in Drosophila melanogaster. Science 221: 23-29.

Cabrera, C.V., J. Botas, and A. Garcia-Bellido. 1985. Distribution of Ultrabithorax proteins in mutants of Drosophila bithorax complex and its transregulatory genes. Nature 318: $569-571$.

Carroll, S.B., R.A. Laymon, M.A. McCutcheon, P.D. Riley, and M.P. Scott. 1986. The localization and regulation of Antennapedia expression in Drosophila embryos. Cell 47: 113122.

Casanova, J., E. Sanchez-Herrero, and G. Morata. 1985. Prothoracic transformation and functional structure of the Ultrabithorax gene of Drosophila. Cell 42: 663-669.

Casanova, J., E. Sanchez-Herrero, A. Busturia, and G. Morata. 1987. Double and triple mutant combinations of the bithorax complex of Drosophila. EMBO I. (in press).

Duncan, I. 1987. The bithorax complex. Annu. Rev. Genet. 21: (in press).

Gehring, W.J. 1987. Homeoboxes in the study of development. Science 236: 1245-1252.

Gelbart, W.M., V.F. Irish, R.D. St. Johnston, F.M. Hoffmann, R.K. Blackman, D. Segal, L.M. Posakony, and R. Grimaila.
1985. The decapentaplegic gene complex in Drosophila melanogaster. Cold Spring Harbor Symp. Quant. Biol. 50: 119-125.

Hafen, E., M. Levine, and W.J. Gehring. 1984. Regulation of Antennapedia transcript distribution by the bithorax complex in Drosophila. Nature 307: 287-289.

Hayes, P.H., S. Sato, and R.E. Denell. 1984. Homeosis in Drosophila; the Ultrabithorax larval syndrome. Proc. Natl. Acad. Sci. 81: 545-549.

Kaufman, T.C., R. Lewis, and B. Wakimoto. 1980. Cytogenetic analysis of chromosome 3 in Drosophila melanogaster: The homeotic gene complex in polytene interval 84A-B. Genetics 94: 115-133.

Karch, F., B. Weiffenbach, M. Peifer, W. Bender, I. Duncan, S. Celniker, M. Crosby; and E.B. Lewis. 1985. The abdominal region of the bithorax complex. Cell 43: 81-96.

Kuner, J.M., M. Nakanishi, Z. Ali, B. Drees, E. Gustavson, J. Theis, L. Kauvar, T. Kornberg, and P.H. O'Farrell. 1985. Molecular cloning of engrailed: A gene involved in development of pattern in Drosophila melanogaster. Cell 42: 309316.

Laughon, A. and M.P. Scott. 1984. Sequence of a Drosophila segmentation gene: Protein structure homology with DNAbinding proteins. Nature 310: $25-31$.

Lewis, E.B. 1963. Genes and developmental pathways. Am. Zool. 3: 33-56.

- 1978. A gene complex controlling segmentation in Drosophila. Nature 276: 565-570.

Lipshitz, H.D., D.A. Peattie, and D.S. Hogness. 1987. Novel transcripts from the Ultrabithorax domain of the bithorax complex. Genes Dev. 1: 307-322.

Martinez-Arias, A. and P. Lawrence. 1985. Parasegments and compartments in the Drosophila embryo. Nature 313: 639642.

Morata, G., J. Botas, S. Kerridge, and G. Struhl. 1983. Homeotoic transformations of the abdominal segments of Drosophila caused by breaking or deleting a central portion of the bithorax complex. J. Embryol. Exp. Morphol. 78: 319-341.

Nüsslein-Volhard, C. and E. Wieschaus. 1980. Mutations affecting segment number and polarity in Drosophila. Nature 287: 795-801.

Peifer, M. and W. Bender. 1986. The anterobithorax and bithorax mutations of the bithorax complex. EMBO $J$. 5: 2293-2303.

Regulaski, M., K. Harding, R. Kostriken, F. Karch, M. Levine, and W. McGinnis. 1985. Homeobox genes of the Antennapedia and bithorax complexes of Drosophila. Cell 43: 7180.

Sanchez-Herrero, E., I. Vernos, R. Marco, and G. Morata. 1985. Genetic organization of the Drosophila bithorax complex. Nature 313: $108-113$.

Scott, M.P., A.J. Weiner, B.A. Polisky, T.I. Hazelrigg, V. Pirrotta, F. Scalenghe, and T.C. Kaufman. 1983. The molecular organization of the Antennapedia complex of Drosophila. Cell 35: 763-776.

Struhl, G. 1984. Splitting the bithorax complex of Drosophila. Nature 308: 454-457.

Struhl, G. and R.A.H. White. 1985. Regulation of the Ultrabithorax gene of Drosophila by other bithorax complex genes. Cell 43: 507-519.

Tiong, S., L.M. Bone, and J.R.S. Whittle. 1985. Recessive lethal mutations within the bithorax-complex in Drosophila. Mol. Gen. Genet. 200: 335-342.

White, R.A.H. and M.E. Akam. 1985. Contrabithorax mutations cause inappropriate expression of Ultrabithorax products in Drosophila. Nature 318: 567-569. 
Peifer et al.

White, R.A.H. and M. Wilcox. 1984. Protein products of the bithorax complex in Drosophila. Cell 39: 163-171.

- 1985a. Distribution of Ultrabithorax protein in Drosophila. EMBO I. 4: 2035-2043.

- 1985b. Regulation of the expression of Ultrabithorax proteins in Drosophila. Nature 318: 563-567.

Wirz, J., L.I. Fessler, and W.J. Gehring. 1986. Localization of the Antennapedia protein in Drosophila embryos and imaginal discs. EMBO J. 5: 3327-3334. 


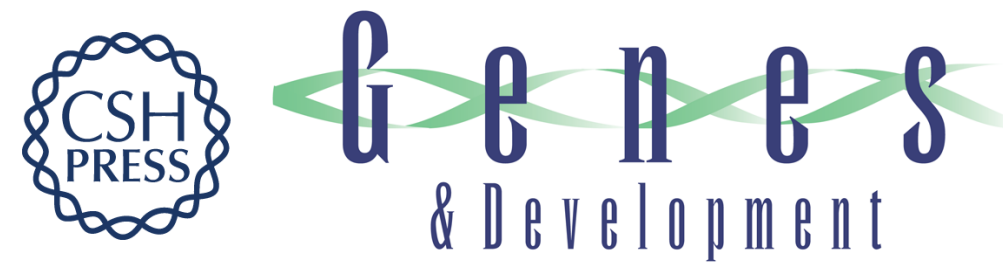

\section{The bithorax complex: control of segmental identity}

Genes Dev. 1987, 1:

Access the most recent version at doi:10.1101/gad.1.9.891

References This article cites 33 articles, 7 of which can be accessed free at: http://genesdev.cshlp.org/content/1/9/891.full.html\#ref-list-1

License

Email Alerting Receive free email alerts when new articles cite this article - sign up in the box at the top Service right corner of the article or click here.

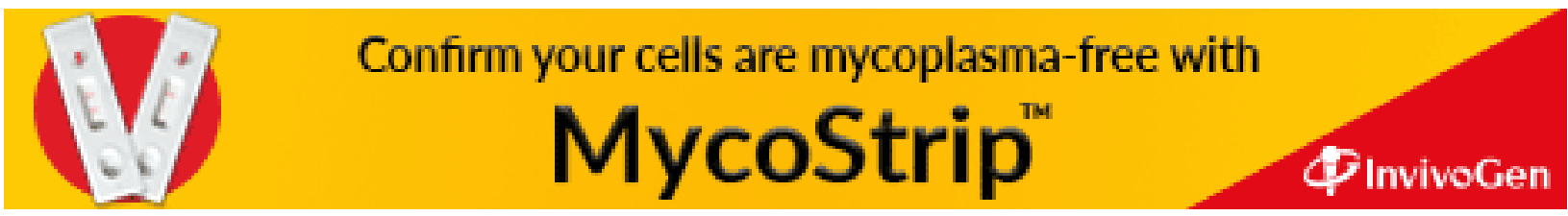

